\title{
A 1-D Granular Gas As A Knudsen Gas ${ }^{1}$
}

\author{
Patricio Cordero*, José Miguel Pasini*, and Rosa Ramírez ${ }^{\dagger}$ \\ * Departamento de Física, Universidad de Chile, Santiago, Chile \\ † CECAM, ENS-Lyon, 46 Allée d'Italie, 669007, France
}

\begin{abstract}
We study a one-dimensional system of inelastic hard particles driven by thermal walls. For low dissipation in collisions the system may be treated as a perturbation on a non-interacting system, being thus conceptually similar to a Knudsen gas. When the quasielastic system is subjected to gravity a kinetic theory approach is suitable, giving a faithful description of results stemming from Newtonian molecular dynamics simulations. Effects derived from the finite size of the particles may be taken into account without invalidating the rarefied system assumption. When the system is not subject to gravity and held between two thermal walls, the boundary conditions determine whether the system will be fluidized or clusterized. The transition from the clustering regime to the fluidized state is described.
\end{abstract}

\section{INTRODUCTION}

Granular systems are many-particle systems characterized by dissipative collisions. When subjected to a sufficiently strong excitation, these systems may behave fluid-like [1-4]. From the very beginning several authors have attempted to derive hydrodynamic equations for these systems [5,6]. A more recent granular hydrodynamics is in [7]. If the excitation of the granular system is through a permanent energy injection, the fluid may stabilize in a low-density stationary gaseous state. This state is necessarily a nonequilibrium state and it usually is inhomogeneous as well. To develop the basic features of the theory of gaseous granular systems we restrict the analysis to the simplifying inelastic hard-sphere model (IHS) [5].

In this work we study a non-trivial dissipative system: the one dimensional system of inelastic hard spheres. This simple model shares some of the phenomena of higher dimensional dissipative systems, such as transition from a condensed to a fluid state [8] and inelastic collapse [9]. Hydrodynamics and kinetic theory have been derived for such systems, when free of clustering, basically from the associated Boltzmann equation [10-12], but there is a crucial fact which makes the 1-D case special: the velocity distribution function is heavily influenced by the boundary conditions. Therefore some of the 1-D results we present here could qualitatively be extended to higher dimensions for Knudsen gases, or even standard gases near the boundaries.

In this paper we study a 1-D system of $N$ point-like particles interacting via collisions (with and without gravity present) that conserve momentum but dissipate kinetic energy. We do theory and Newtonian molecular dynamic simulations. The inelastic collision rules which take the velocities $\left(c_{1}, c_{2}\right)$ into $\left(c_{1}^{\prime}, c_{2}^{\prime}\right)$ are

$$
c_{1}^{\prime}=q c_{1}+(1-q) c_{2}, \quad c_{2}^{\prime}=q c_{2}+(1-q) c_{1},
$$

where $0 \leq q \leq 1 / 2$, and $q=0$ corresponds to the perfectly elastic case. The usual restitution coefficient is $r=1-2 q$. Our study was originally focused on describing stationary gaseous states of the system but, as we will see, there are circumstances when the system develops a cluster and does not settle to a stationary state.

If particles are relabeled after each collision, so that in (1), $c_{1}^{\prime} \longleftrightarrow c_{2}^{\prime}$ it is seen that the limit $q=0$ is tantamount to a system of $N$ point particles that pass through each other without ever interacting. In the case $q \neq 0$ particles pass through each other losing some energy in the process, as if moving through a viscous background.

The limit $q=0$ makes the system trivial, in the sense that particles do not interact with each other, and Liouville's problem becomes a one-free-particle problem. The exact kinetic equation becomes Boltzmann's

1) URL: http://www.cec.uchile.cl/cinetica/ 
equation itself with a vanishing collision term because Liouville's distribution is the simple product of $N$ oneparticle distribution functions. The inelastic case, with $q$ sufficiently small, can be regarded as a perturbation of the free case. It has been seen that a true perturbation theory can be defined when the parameter is not simply $q$ but the product $q N$ with $N$ arbitrarily large [12], all this assuming that no clusters are formed.

To achieve gaseous states a permanent energy injection is needed, which we have chosen to be a thermal wall. Namely, a particle hitting this wall bounces back as if coming from a heat bath at temperature $T_{\text {wall }}$.

Our work has focused on the study of two different regimes. On one hand, stationary states can be found when the dissipation rate in the system is sufficiently low. In this case we show that a theoretical treatment based on Boltzmann's equation is suitable for describing the system. On the other hand, at high dissipation rates, clusters may form in the system. In this case, the kinetic theory approach mentioned is not suitable anymore, and simulational studies are needed for developing insight into this regime. As we will see, the dissipation rate depends both on the inelastic coefficient $q$ as well as on the boundary conditions and any external field applied to the system.

The 1-D Boltzmann equation in the inelastic point particle case with $\int f(x, c) d x d c=1$ and precollision velocities $c_{1}^{*}=\left[(1-q) c_{1}-q c_{2}\right] /(1-2 q) \quad$ and $\quad c_{1}^{*}=\left[(1-q) c_{2}-q c_{1}\right] /(1-2 q)$ is

$$
\left(\frac{\partial}{\partial t}+c \frac{\partial}{\partial x}-g \frac{\partial}{\partial c}\right) f(x, c, t)=N \int\left(\frac{1}{(1-2 q)^{2}} f\left(x, c_{1}^{*}, t\right) f\left(x, c_{2}^{*}, t\right)-f(x, c, t) f\left(x, c_{2}, t\right)\right)\left(c-c_{2}\right) d c_{2}
$$

If $q=0$ the right hand side can be shown to vanish. Restricting our study to the limit $N \rightarrow \infty$, but keeping $q N$ finite, the equation reduces to [12]

$$
\begin{aligned}
\left(\frac{\partial}{\partial t}+c \frac{\partial}{\partial x}-g \frac{\partial}{\partial c}\right) f(x, c, t) & =q N \frac{\partial}{\partial c}[f(x, c, t) M(x, c, t)] \\
M(x, c, t) & \equiv \int\left|c-c^{\prime}\right|\left(c-c^{\prime}\right) f\left(x, c^{\prime}, t\right) d c^{\prime}
\end{aligned}
$$

A method of solving this equation is by expanding the distribution in powers of $q N$ and solving consistently at each order.

If the system consists of rods of size $\sigma$ instead of point-like particles the situation changes radically. The systems proceeds via inelastic hard collisions (1) exactly through the same set of velocity states, provided the box is enlarged from $L$ to $L^{\prime}=L+N \sigma$. In this respect the velocity distribution alone is the same, but one cannot describe the elastic limit as equivalent to particles passing through each other without interacting. If particles are still relabeled after each collision, they will make instantaneous jumps of length $\sigma$ at every collision. If again the passage from Liouville's equation to a one particle equation is attempted, the approximate equation will have a non-trivial collision term. Some configurational correlations exist and they have to be described using an Enskog-type equation [14]. The most obvious differences are obtained looking at the effects of $\sigma$ on the density profile, as seen later on.

Finally, we focus our study of the transition from the clustering regime to fluidized states by simulating a system of point particles not subject to gravity between two thermal walls at different temperatures $T_{\text {left }}$ and $T_{\text {right. }}$ For this problem, when

$$
\Delta \equiv \frac{T_{\text {right }}-T_{\text {left }}}{T_{\text {right }}+T_{\text {left }}}
$$

is below a certain threshold, a unique cluster is formed and the system remains in a time-dependent state that we describe further on and of which a detailed account is given elsewhere [13].

\section{SYSTEM IN AN OPEN BOX UNDER THE ACTION OF GRAVITY}

\section{Point-like particles}

In this case (grains of mass $m=1$, base at temperature $T_{\text {base }}$ in energy units, and acceleration of gravity $g$ ) there is no cluster formation in the quasielastic case, and Boltzmann's equation (2) can be applied with no restriction. Equation (3) is solved perturbatively and it gives an extraordinarily faithful description of the 

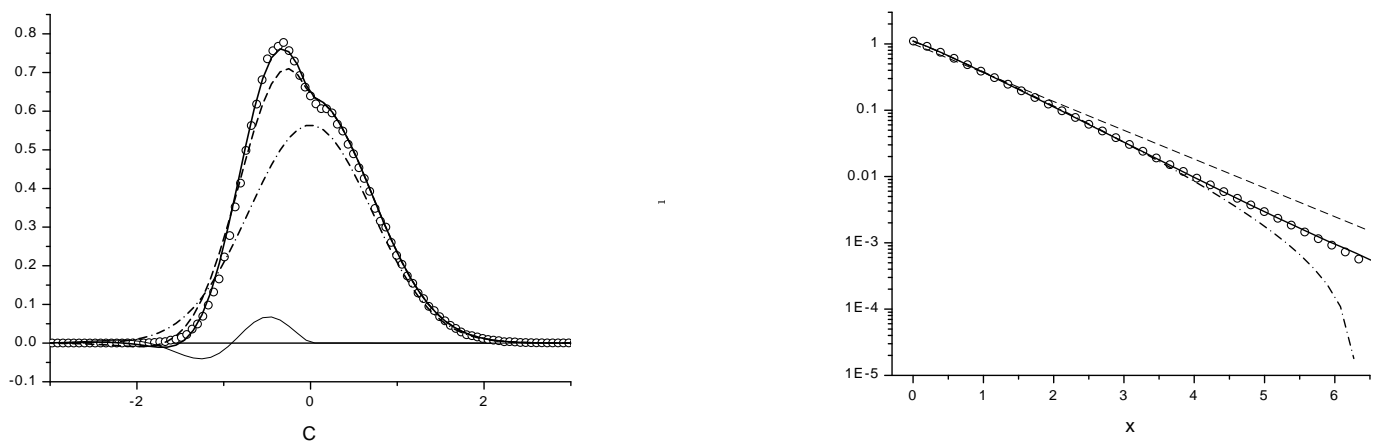

FIGURE 1. At left is the distribution function at the base $(x=0)$ normalized to 1 evaluated to zeroth (dot-dashed line), first (dashed line) and second order (solid line) for $q N=0.2$ and $N=200$. The observed distribution differs considerably from the zeroth order approximation. In spite of this, the second order approximation already matches extraordinarily well the simulational data (open circles). The solid S shaped curve underneath represents the second order correction. Notice that this correction is almost zero for positive velocities. At right circles show in a logarithmic scale the density observed in a simulation of a system with $q N=0.1$ and $N=200$. The density is shown evaluated up to zero (dashed line), first (dot-dash line) and second order (solid line). It can be seen that the prediction up to second order reproduces extraordinarily well the density at heights few particles reach.

behavior of the simulated system when $q N$ is not too large. Figure 1 shows the velocity distribution function at the base. Usually predicting the shape of the distribution at a wall is harder than getting it far from the walls but the perturbation method is powerful enough. The abrupt change in the behavior of the distribution at the base about $c=0$ can be understood because the particles near the base and going up are dominated by the thermal boundary condition. Hence they have almost no information from the bulk and they are described by a distribution close to a Maxwellian, while on the other hand the particles arriving to the base carry the influence of their passage through the bulk. These two distributions meet at the origin of phase-space producing the singular behaviour seen at left in Fig. 1.

Defining the characteristic height $h_{c}$ of the system as the height below which, on the average, $90 \%$ of the particles are found, it will be seen that the present theoretical framework gives a good description well beyond $h_{c}$. This height depends on $q N$ but in the range $0 \leq q N \leq 0.2$ it varies from 2.7 to 1.65 (in units of $T_{\text {base }} / g$ ) hence taking $h_{c} \approx 2$ as the characteristic length in all our cases is reasonable. Figure 1 also shows, at right, the density of the system up to a height $x=6$, where the density is three orders of magnitud smaller than at the base, and still the second order perturbative solution fits well the observed density.

The velocity of particles falling through a viscous fluid tends to a limiting value. The meaning of the maximum that the velocity distribution has for $c<0$ at $x=0$ seems to be related to a similar phenomenon even though our fluid cannot be described as a fluid of uniform viscosity.

We used a system of $N=200$ particles because, as suggested at left in Fig. 2, which shows the temperature profile for systems of different size (up to $N=1000$ ), size independence is already reached for $N=200$ when $q N \leq 0.1$. Units are such that $T=0.5$ for the perfectly elastic system.

\section{A gas of rods}

If the system consists of rods of size $\sigma$ an Enskog-type kinetic equation has to be used to consider that colliding particles are in different positions and that there are excluded-volume effects. The size effects (measured with $\zeta=\sigma N g / T_{\text {wall }}$ ) compete with the inelastic effects (measured with $q N$ ) and it is interesting to look at regimes where they are somehow comparable. The results presented in Fig. 3 in this subsection correspond to cases 

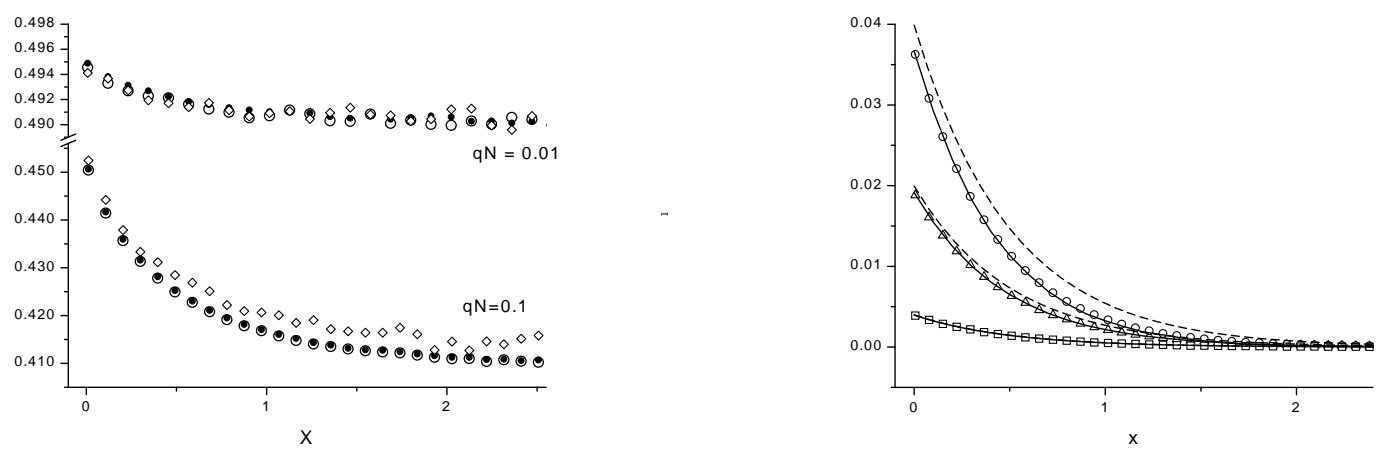

FIGURE 2. At left the temperature profile, observed in systems with $q N=0.01$ (upper set of points) and with $q N=0.1$ (lower set of points) for systems with $N$ particles: $N=20$ (rhombus), $N=200$ (solid circles), and $N=1000$ (open circles). For $q N=0.1$ the profiles with $N=200$ and $N=1000$ coincide because the size independent behavior has been reached while the points for $N=20$ show a higher temperature. For $q N=0.01$ even for $N=20$ the asymptotic behavior of the temperature has been reached bacause second order corrections (where the size appears for the first time) are too small. At right the permanent energy influx through the base is dissipated in the "volume". The figure shows the heat flux $J_{Q}$, as a function of height for systems with $N=200$ and $q N=0.01$ (squares), $q N=0.05$ (triangles) and $q N=0.1$ (circles). The solid (dashed) lines give the respective heat fluxes evaluated up to second (first) order.
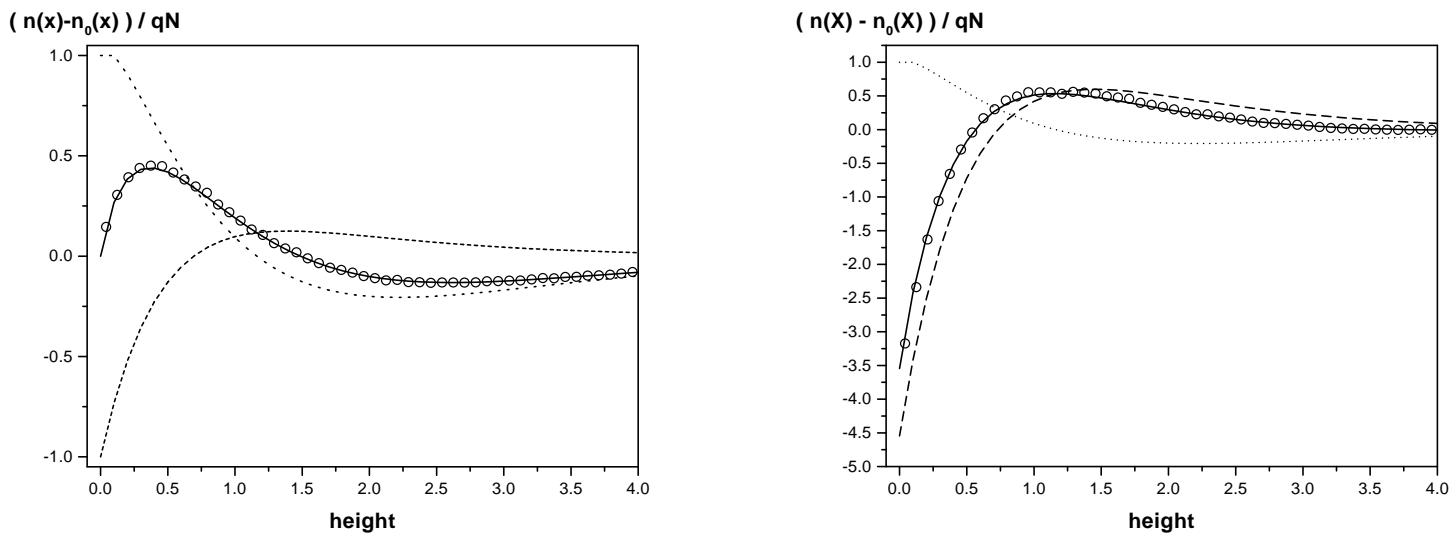

FIGURE 3. At left the profile of the density correction $\frac{1}{q N}\left(n-n_{0}\right)$ as a function of height for a system with $\zeta=0.1$ and $q N=0.02$. The dotted line considers only the $\zeta$ corrective term (up to second order in $\zeta$ ), the dashed line considers only the dissipative correction up to first order in $q N$. The solid line shows the combined effect while the circles are the data obtained from the molecular dynamic simulations. At right a system with $\zeta=q N=0.02$ with both corrections evaluated up to first order. Notice that the density correction cannot be too large near the base because of the size of the rods. In both cases theory fits excellently the simulational data.

in which the effects of dissipation were evaluated up to first order [14]. It is possible to check that the pair correlation function has a non-trivial structure even in the purely elastic case [15].

Here comes into play one distinctive feature of the one-dimensional system: taking into account corrections due to the finite (but small) size of the particles is not inconsistent with the rarefied system assumption. 


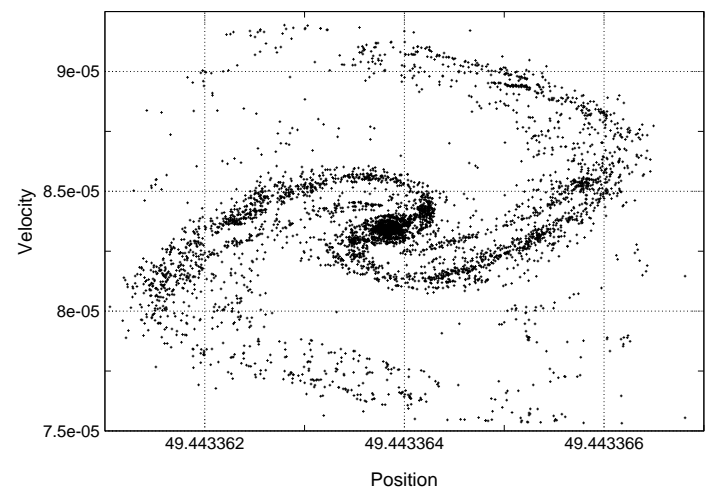

FIGURE 4. A cluster seen in phase space. The system consists of $N=10000$ particles, $q N=0.1$ and the temperature at both walls is the same. This particular cluster contains approximately half the particles of the system and the size of the cluster is about $2.5 \times 10^{-5}$ the size of the linear box.

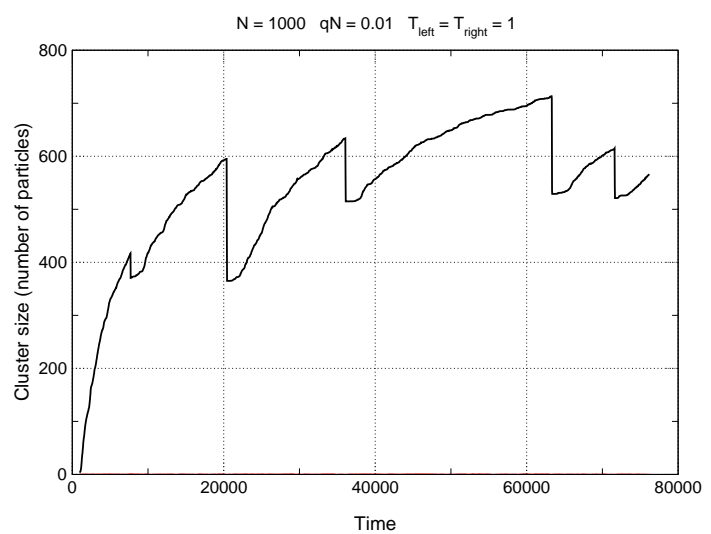

FIGURE 5. The cluster grows continuously with time until it hits a wall. The collision provokes the evaporation of part of its particles, this vapor pushes the cluster away from the wall and the growing process is resumed.

Therefore the system may be treated as a perturbation to the non-collisional case. By expanding the collision term in Boltzmann's equation in a double series in $q N$ and $\zeta$ [14] we can consider to a different extent size and dissipation effects.

Figure 3 compares the density corrections due to dissipation and to the finite size of the particles. It can be seen that at the base these effects compete: dissipation tends to increase the density, while the size of the particles tends to decrease it. The combined effect matches closely the results obtained from simulations.

\section{THE GAS OF INELASTIC POINTS IN THE ABSENCE OF GRAVITY}

\section{Presence of a cluster}

The 1-D system is left to evolve between two thermal walls at temperatures $T_{\text {left }}$ and $T_{\text {right }}$. We choose $T_{\text {left }}<T_{\text {right }}$ and units such that $T_{\text {left }} T_{\text {right }}=1$. The parameter $\Delta$ defined in (4) is used to quantify how far from the symmetrical case $(\Delta=0)$ is the system. The case $\Delta=1$ represents an infinitely strong temperature gradient.

Our simulations show that for $\Delta=0$ a cluster unavoidably forms and moves at random about the center of the box. If $\Delta \neq 0$ but small a cluster still forms, but it roams closer to the colder wall. In molecular dynamics simulations we detect clusters by looking for chains of particles two or three orders of magnitud closer than 
$\frac{1}{N}$ times the size of the box. Figure 4 shows the tiny portion of phase space where the cluster is concentrated. The spiral shape is time-dependent, as the cluster continues to slowly contract.

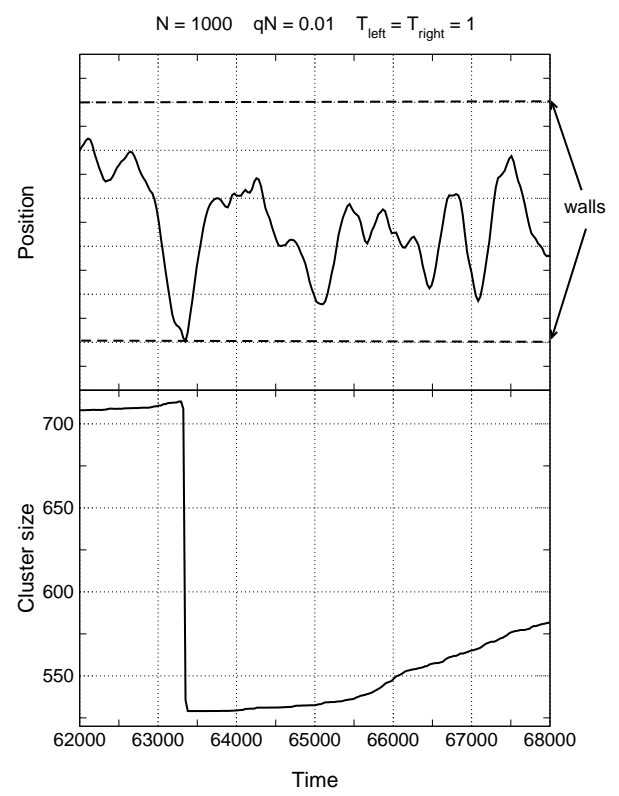

FIGURE 6. At top the cluster's position as a function of time sometime before hitting a wall and the evolution afterwards. At bottom the evolution of the size of the cluster in the same time lapse.

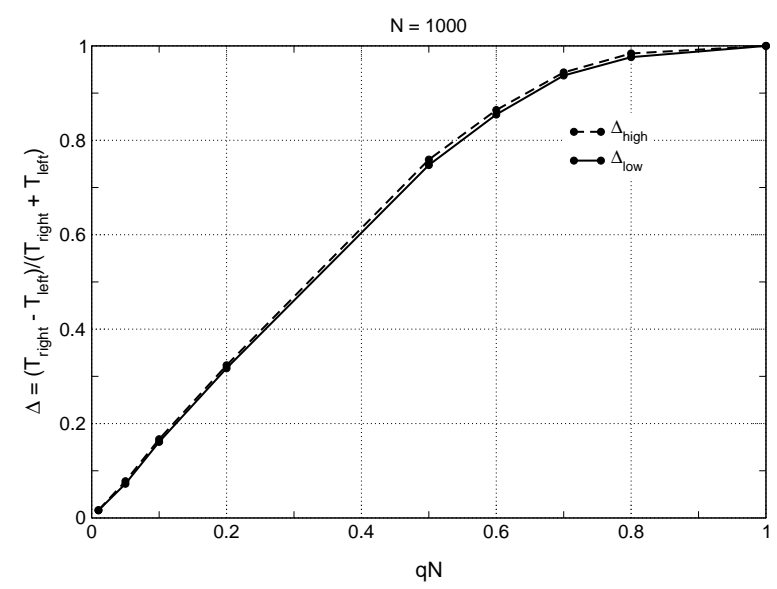

FIGURE 7. Clustering transition: when $\Delta$ is above the threshold shown in the figure the system does not exhibit cluster formation and is completely fluidized. The zone between the two curves represents a regime where it is difficult to decide whether clusters will form.

Whenever a cluster exists it keeps growing in size. This makes the rest of the system more rarefied in time, so that a cluster-wall collision becomes more probable. Figure 5 shows the size of the cluster (in number of particles) as a function of time. It is seen that there are sudden size reductions which correspond to the instants when the cluster hits a wall. One of these collisions is shown in detail in Fig. 6. At top the position of the cluster is seen as a function of time showing the exact moment when the collision occurs. At bottom, in the same figure, it is seen how the size of the cluster has a sudden reduction to restart the growth process. Thus, not only is the system highly clumped, but it is in a non-steady state as well. 

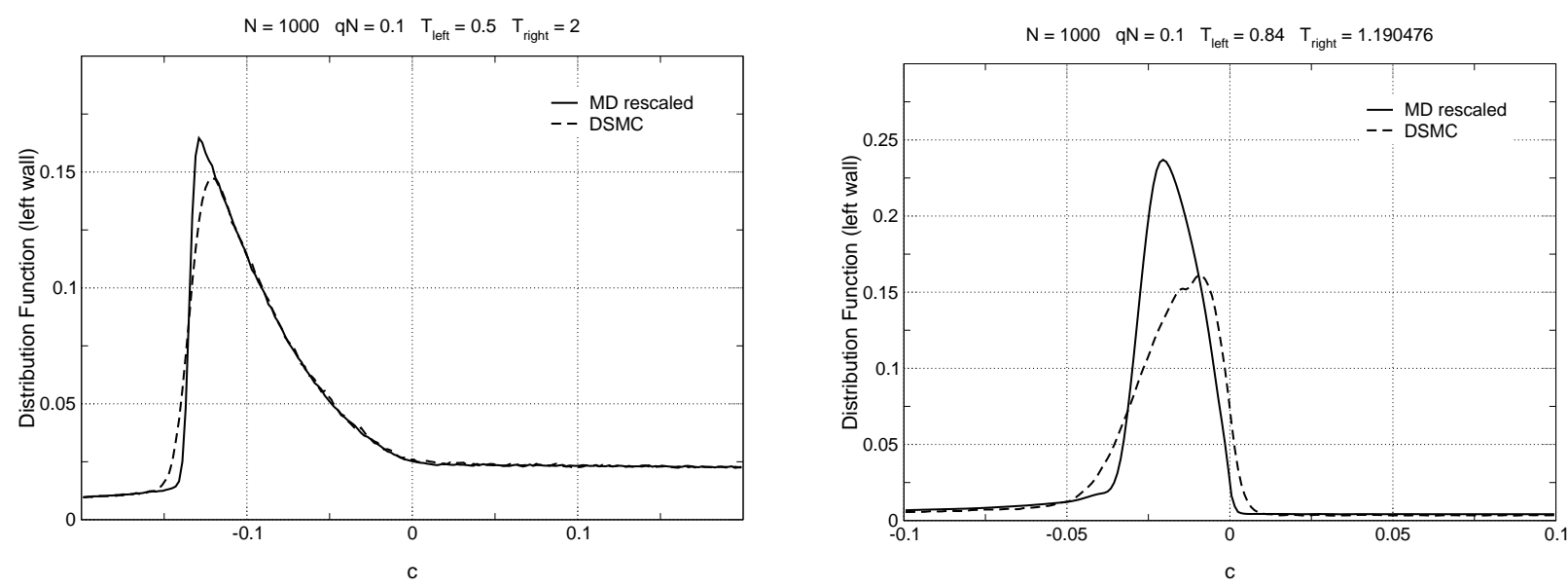

FIGURE 8. The velocity distribution functions at the left wall obtained from Newtonian molecular dynamics (solid lines) are compared with the distributions obtained from DSMC (dashed lines) for a system of $N=1000$ particles and $q N=0.1$. For this value of $q N$ a cluster forms roughly when $\Delta<0.16$. At left the case $\Delta=0.60$ which is far from the threshold and at right the case $\Delta=0.172$.
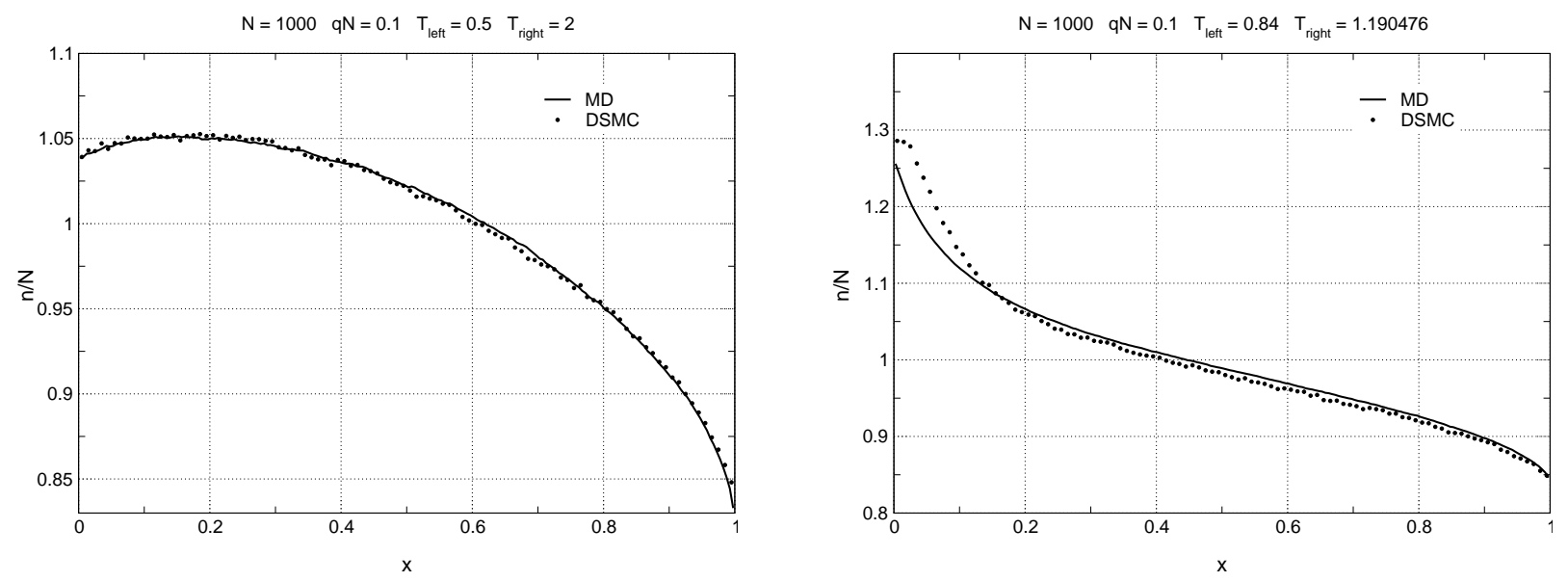

FIGURE 9. Density profiles for the cases depicted in Fig. 8.

\section{The completely fluidized system}

By increasing $\Delta$ the frequency of cluster-wall collisions grows, sometimes obtaining "windows" in which the cluster completely evaporates. By further increasing $\Delta$ these windows grow until a point is reached where no cluster is formed. In this fashion a totally fluidized stationary state is achieved. Figure 7 shows, for $N=1000$ particles, the curve that separates the fluidized from the clustering regime. When $\Delta$ is below but close to the transition curve clustering occurs in windows, while farther from the transition the cluster is always present.

If the state of the system is sufficiently far from the threshold of cluster formation it may be described with the dissipative Boltzmann equation (2). But since the velocity distribution obtained from MD simulations exhibits a peculiar non-Gaussian feature at slow velocities which resembles the singular behavior observed when there is a cluster [13], it may also be that there are still dominant correlation effects that make Boltzmann's equation inapplicable. To discern this we compare results from Newtonian molecular dynamics (MD) with the corresponding results arising from direct-simulation Monte Carlo (DSMC), which neglects correlations. As seen in Figs. 8 and 9, the results agree very well, except when the value of $\Delta$ is close to the onset of clustering. 


\section{CONCLUSIONS}

We have established that a quasielastic 1-D system under gravity may be thought of as a rarefied gas: the collision term of the associated Boltzmann equation may be considered small and the boundary conditions affect the state of the system throughout the volume. Results derived using perturbation theory match well those obtained from molecular dynamics simulations. Effects due to the finite size of the particles may also be considered perturbatively, consistently with the Knudsen gas assumption.

The same system not subject to gravity was also studied. Confined between two walls at different temperatures, fluidized and clustering states are found. The distribution function exhibits distinctive non-Gaussian features. When the system is kept fluidized, these features are shown not to arise from correlations by comparing MD and DSMC results.

Acknowledgments: We would like to thank Rodrigo Soto, Dino Risso, and Aldo Frezzotti for fruitful discussions. This work has been partially funded by Fondecyt through grants 1000884 (P.C.) and 2990108 (J.M.P.). J.M.P. would like to thank Fundación Andes for a doctoral scholarship and R.R. would like to acknowledge a Marie Curie postdoctoral fellowship.

\section{REFERENCES}

1. H. M. Jaeger and S. R. Nagel, Science 255, 1523 (1992); H. M. Jaeger, S. R. Nagel, and R. P. Behringer, Phys. Today 49, 32 (1996); H. M. Jaeger, S. R. Nagel, and R. P. Behringer, Rev. Mod. Phys. 68, 1259 (1996).

2. J. A. C. Gallas, H. J. Herrmann, and S. Sokolowski, Physica A 189, 437 (1992); Y. Zhang and C. S. Campbell, J. Fluid Mech. 237, 541 (1992); S. Luding, H. J. Herrmann, and A. Blumen, Phys. Rev. E 50, 3100 (1994); S. Warr, J. M. Huntley, and G. T. H. Jacques, Phys. Rev. E 52, 5583 (1995); N. Mujica and F. Melo, Phys. Rev. Lett. 80, 5121 (1998).

3. L. P. Kadanoff, Rev. Mod. Phys. 71, 435 (1999).

4. Rosa Ramírez and Patricio Cordero, cond-mat/0002433, to appear in Phys. Rev. Lett.

5. C. S. Campbell, Annu. Rev. Fluid Mech. 22, 57 (1990).

6. J. T. Jenkins and S. B. Savage, J. Fluid Mech. 130, 187 (1983); C. Lun, S. Savage, D. Jeffrey, and R. P. Chepurnuy, J. Fluid Mech. 140, 223 (1984); J. T. Jenkins and M. W. Richman, Arch. Rat. Mech. Anal. 87, 355 (1985); J. T. Jenkins and M. W. Richman, Phys. Fluids 28, 3485 (1985); P. K. Haff, J. Fluid Mech. 134, 401 (1983).

7. Rosa Ramírez, D. Risso, R. Soto, and P. Cordero, cond-mat/0001250, to appear in Phys. Rev. E.

8. E. Clément, S. Luding, A. Blumen, J. Rajchenbach, and J. Duran, Int. J. Mod. Phys. B 7, 1807 (1993); S. Luding, E. Clément, A. Blumen, J. Rajchenbach, and J. Duran, Phys. Rev. E 49, 1634 (1994).

9. S. McNamara and W. R. Young, Phys. Fluids A 4, 496 (1992); S. McNamara and W. R. Young, Phys. Rev. E 50, R28 (1994).

10. N. Sela and I. Goldhirsch, Phys. Fluids 7, 507 (1995).

11. S. McNamara and W. R. Young, Phys. Fluids A 5, 34 (1993).

12. Rosa Ramírez and P. Cordero, Phys. Rev. E 59, 656 (1999).

13. J. M. Pasini and P. Cordero, cond-mat/0006123.

14. Rosa Ramírez, Ph. D. thesis, Universidad de Chile (1998).

15. J. Ibsen, P. Cordero, and R. Tabensky, Phys. Fluids 8, 12 (1997). 